PROCEEDINGS OF THE

AMERICAN MATHEMATICAL SOCIETY

Volume 137, Number 2, February 2009, Pages 563-572

S 0002-9939(08)09682-2

Article electronically published on October 8, 2008

\title{
INTERSECTION OF DILATES OF SHIFT-INVARIANT SPACES
}

\author{
MARCIN BOWNIK
}

(Communicated by Michael T. Lacey)

\begin{abstract}
We prove that if the dimension function of a shift-invariant space $V$ is not constantly $\infty$, then the intersection of (negative) dilates of $V$ must be trivial. We also give an example of two refinable shift-invariant spaces with identical spectral functions such that this intersection is either trivial or non-trivial.
\end{abstract}

\section{INTRODUCTION}

A fundamental concept in the theory of wavelets is the notion of a multiresolution analysis (MRA) formulated by Mallat [14. An MRA is a sequence of closed subspaces $\left\{V_{j}\right\}_{j \in \mathbb{Z}}$ of $L^{2}(\mathbb{R})$ satisfying

$$
\begin{aligned}
& V_{j} \subset V_{j+1} \quad \text { for } j \in \mathbb{Z}, \\
& f \in V_{j} \Longleftrightarrow f(2 \cdot) \in V_{j+1} \quad \text { for } j \in \mathbb{Z}, \\
& \bigcap_{j \in \mathbb{Z}} V_{j}=\{0\}, \\
& \overline{\bigcup_{j \in \mathbb{Z}} V_{j}}=L^{2}(\mathbb{R}),
\end{aligned}
$$

The condition (1.5) on the existence of a scaling function $\varphi$ is often relaxed by the requirement that $V_{0}$ be shift-invariant (SI), i.e.,

$$
T_{k} V_{0}=V_{0} \quad \text { for all } k \in \mathbb{Z},
$$

where $T_{k} f(x)=f(x-k)$. In this case, one obtains a concept of a generalized multiresolution analysis (GMRA) developed by Baggett, Medina, and Merrill [3].

It is well known that every MRA gives rise to a wavelet; see [13, Proposition II.2.13]. Conversely, any mildly regular wavelet $\psi$ is associated with an MRA by a result of Auscher [1, i.e., that the spaces

$$
V_{j}=\overline{\operatorname{span}}\left\{\psi_{i, k}: i<j, k \in \mathbb{Z}\right\}, \quad \text { where } \psi_{i, k}(x)=2^{i / 2} \psi\left(2^{i} x-k\right),
$$

Received by the editors December 20, 2007.

2000 Mathematics Subject Classification. Primary 42C40.

Key words and phrases. Shift-invariant space, refinable space, the dimension function, the spectral function, GMRA.

The author was partially supported by NSF grant DMS-0653881.

(C)2008 American Mathematical Society Reverts to public domain 28 years from publication 
form an MRA. Moreover, a general orthonormal wavelet $\psi$ is always associated with a GMRA. Furthermore, the class of GMRAs giving rise to a wavelet was characterized in [3] in terms of a consistency equation.

Once we consider a larger class of Parseval wavelets the situation becomes more complex. Recall that $\psi \in L^{2}(\mathbb{R})$ is a Parseval wavelet if

$$
\|f\|^{2}=\sum_{j, k \in \mathbb{Z}}\left|\left\langle f, \psi_{j, k}\right\rangle\right|^{2} \quad \text { for all } f \in L^{2}(\mathbb{R}) .
$$

An unresolved problem of Baggett asks whether every Parseval wavelet is associated with a GMRA. For the current state of this problem we refer to [8]. Here, we only remark that the spaces (1.7) are known to satisfy all properties of a GMRA with the hypothetical exception of (1.3). In fact, the intersection property (1.3) could fail spectacularly for non-tight frame wavelets; see [10]. The goal of this paper is to give a natural sufficient condition for (1.3) to hold.

Suppose that $V$ is an SI space. We say that $V$ is refinable if $V \subset D(V)$, i.e., if (1.1) and (1.2) hold for $V_{j}=D^{j}(V)$. Here, $D$ is the dyadic dilation operator given by $D f(x)=\sqrt{2} f(2 x)$. If $V$ is refinable, then the intersection property (1.3) can be written as

$$
\bigcap_{j=-\infty}^{0} D^{j}(V)=\{0\}
$$

It was proved in [13, Theorem II.1.6] that if $V$ is singly generated, then (1.8) holds. For a shift-invariant space singly generated by $\varphi \in L^{2}(\mathbb{R})$, its dimension function is the characteristic function of the union of integer translates of the support of $\hat{\varphi}$. Since a general shift-invariant space $V$ is an orthogonal sum of singly generated spaces, one can extend the notion of dimension function using its additivity on such sums. Rzeszotnik [15] proved that (1.8) holds if the dimension function $\operatorname{dim}_{V}$ of $V$ is locally integrable. The main result of this paper shows that (1.8) holds if merely $\operatorname{dim}_{V}$ is not constantly $\infty$.

A similar result for a non-stationary sequence of PSI spaces $\left\{V^{(j)}\right\}_{j \in \mathbb{N}}$ (each space has 1 generator) is due to de Boor, DeVore, and Ron 44. Indeed, by 4, Theorem 4.9] the intersection $\bigcap_{j \in \mathbb{Z}} D^{j}\left(V^{(j)}\right)$ is at most 1-dimensional. In the case when each space $V^{(j)}$ has $L$ generators, the intersection has dimension $\leq L$; see [7, Theorem 3.5].

Despite that our work is motivated by the classical setting of dyadic dilations, our main result holds for much more general sequences of dilations.

Theorem 1.1. Suppose $V \subset L^{2}\left(\mathbb{R}^{n}\right)$ is an SI space such that $\operatorname{dim}_{V}$ is not identically $\infty$ a.e. Let $\left\{A_{j}\right\}_{j \in \mathbb{N}}$ be a sequence of invertible $n \times n$ matrices such that

$$
\left\|A_{j}\right\| \rightarrow 0 \quad \text { as } j \rightarrow \infty \text {. }
$$

Then,

$$
\bigcap_{j=1}^{\infty} D_{j}(V)=\{0\}, \quad \text { where } D_{j} f(x)=f\left(A_{j} x\right) .
$$

The paper is organized as follows. In the next section we state necessary results about the spectral function of SI spaces and give the proof of Theorem 1.1. In the last section we give examples of two SI spaces illustrating subtleties when dealing with the intersection property (1.8). 


\section{Preliminaries and the proof of the main Result}

We start by recalling some rudimentary facts about SI spaces.

Definition 2.1. We say that a closed subspace $V \subset L^{2}\left(\mathbb{R}^{n}\right)$ is shift-invariant (SI) if $T_{k} V \subset V$ for all $k \in \mathbb{Z}^{n}$. Suppose that $\mathcal{A} \subset L^{2}\left(\mathbb{R}^{n}\right)$ is countable. The SI space generated by $\mathcal{A}$ is

$$
\mathcal{S}(\mathcal{A})=\overline{\operatorname{span}}\left\{T_{k} \varphi: \varphi \in \mathcal{A}, k \in \mathbb{Z}^{n}\right\} .
$$

In the case when $\mathcal{A}$ consists of a single function $\varphi \in L^{2}\left(\mathbb{R}^{n}\right)$, we say that $\mathcal{S}(\mathcal{A})=$ $\mathcal{S}(\varphi)$ is a principal shift-invariant (PSI) space.

We will need the following fact about PSI spaces; see [5].

Lemma 2.1. Suppose $V=\mathcal{S}(\varphi)$ is a PSI space. Then, $f \in V$ if and only if $f \in L^{2}\left(\mathbb{R}^{n}\right)$ and

$$
\hat{f}(\xi)=m_{f}(\xi) \hat{\varphi}(\xi) \quad \text { for a.e. } \xi \in \mathbb{R}^{n}
$$

for some $\mathbb{Z}^{n}$-periodic measurable function $m_{f}$. Here, the Fourier transform is defined by

$$
\hat{f}(\xi)=\int_{\mathbb{R}^{n}} f(x) e^{-2 \pi i\langle x, \xi\rangle} d x .
$$

The dimension function of an SI space $V=\mathcal{S}(\mathcal{A})$ is a mapping $\operatorname{dim}_{V}: \mathbb{R}^{n} \rightarrow$ $\mathbb{N} \cup\{0, \infty\}$ defined as

$$
\operatorname{dim}_{V}(\xi)=\operatorname{dim} J(\xi), \quad \text { where } J(\xi)=\overline{\operatorname{span}}\{\mathcal{T} \varphi(\xi): \varphi \in \mathcal{A}\} .
$$

Here, $\mathcal{T}$ is a fiberization map given by

$$
\mathcal{T} f(\xi)=(\hat{f}(\xi+k))_{k \in \mathbb{Z}^{n}}
$$

The operator $\mathcal{T}$ is an isometric isomorphism between $L^{2}\left(\mathbb{R}^{n}\right)$ and the vectorvalued space $L^{2}\left(\mathbb{T}^{n}, \ell^{2}\left(\mathbb{Z}^{n}\right)\right)$, where $\mathbb{T}^{n}$ is identified with its fundamental domain $I_{n}=[-1 / 2,1 / 2)^{n}$. The fact that the definition of $\operatorname{dim}_{V}$ is independent of the choice of generators $\mathcal{A}$ follows from the characterization of SI spaces in terms of range functions, see [4, 6], which can be traced back to the work of Helson [12]. Alternatively, $\operatorname{dim}_{V}$ can be defined as the multiplicity function of the projection-valued measure coming from the representation of $\mathbb{Z}^{n}$ on $V$ via translations by Stone's Theorem [2, 3.

More interestingly for us, the dimension function can also be expressed in terms of the spectral function introduced by Rzeszotnik and the author [9]. The same object was unknowingly rediscovered by $\mathrm{Gu}$ and Han under the name "modular function" in [11. While there exist several equivalent ways of defining the spectral function of an SI space $V$, the following result may also serve as a definition; see [9, Proposition 2.2].

Theorem 2.1. Let $\mathfrak{S}$ be the set of all SI subspaces of $L^{2}\left(\mathbb{R}^{n}\right)$. Then, the spectral function $\sigma_{V}$ of $V \in \mathfrak{S}$ is determined as the unique mapping

$$
\sigma: \mathfrak{S} \rightarrow L^{\infty}\left(\mathbb{R}^{n}\right),
$$

such that:

1. $\sigma$ is additive with respect to the orthogonal sums, i.e.,

$$
V=\bigoplus_{i \in \mathbb{N}} V_{i} \quad \text { for some } V_{i} \in \mathfrak{S} \Longrightarrow \sigma_{V}=\sum_{i \in \mathbb{N}} \sigma_{V_{i}}
$$


2. if $V=\mathcal{S}(\varphi)$ is a principal SI space, then

$$
\sigma_{V}(\xi)= \begin{cases}|\hat{\varphi}(\xi)|^{2}\left(\sum_{k \in \mathbb{Z}^{n}}|\hat{\varphi}(\xi+k)|^{2}\right)^{-1} & \text { for } \xi \in \operatorname{supp} \hat{\varphi}, \\ 0 & \text { otherwise. }\end{cases}
$$

Since every SI space $V$ can be decomposed into an orthogonal sum of at most countable PSI spaces [6], Theorem 2.1 provides a convenient way for computing $\sigma_{V}$. Moreover, the result of this computation is independent of the choice of such a decomposition. Finally, we list some of the properties of the spectral function.

1. For any SI space $V$, its spectral function $\sigma_{V}(\xi)$ takes values in $[0,1]$ for a.e. $\xi$.

2. The dimension function of $V$ can be computed by

$$
\operatorname{dim}_{V}(\xi)=\sum_{k \in \mathbb{Z}^{n}} \sigma_{V}(\xi+k) \quad \text { for a.e. } \xi \in \mathbb{R}^{n} .
$$

3. If $V$ and $W$ are two SI spaces such that $V \subset W$, then $\sigma_{V} \leq \sigma_{W}$.

4. If

$$
V=\check{L}^{2}(E)=\left\{f \in L^{2}\left(\mathbb{R}^{n}\right): \operatorname{supp} \hat{f} \subset E\right\}
$$

for some measurable set $E \subset \mathbb{R}^{n}$, then $\sigma_{V}=\mathbf{1}_{E}$. Conversely, if $\sigma_{V}=\mathbf{1}_{E}$, then $V=\check{L}^{2}(E)$.

We are now ready to give the proof of our main result.

Proof of Theorem 1.1. On the contrary, suppose there exists $0 \neq \psi \in L^{2}\left(\mathbb{R}^{n}\right) \in$ $D_{j}(V)$ for all $j \in \mathbb{N}$. Hence, each of the functions $\psi_{j}=\left(D_{j}\right)^{-1} \psi$ belongs to $V$. Note that

$$
\hat{\psi}_{j}(\xi)=\left|\operatorname{det} B_{j}\right| \hat{\psi}\left(B_{j} \xi\right), \quad \text { where } B_{j}=\left(A_{j}\right)^{T} .
$$

By Theorem 2.1 the spectral function of $\mathcal{S}\left(\psi_{j}\right)$ equals

$$
\sigma_{j}(\xi):=\sigma_{\mathcal{S}\left(\psi_{j}\right)}(\xi)=\frac{\left|\hat{\psi}\left(B_{j} \xi\right)\right|^{2}}{\sum_{k \in \mathbb{Z}^{n}}\left|\hat{\psi}\left(B_{j}(\xi+k)\right)\right|^{2}} \quad \text { for } \xi \in \operatorname{supp} \hat{\psi}_{j} .
$$

Since $\mathcal{S}\left(\psi_{j}\right) \subset V$, the monotonicity of the spectral function implies that

$$
\sigma_{V}(\xi) \geq \sigma_{j}(\xi) \quad \text { for all } j \in \mathbb{N} .
$$

For a fixed $N>0$, define the functions

$$
L_{j}(\xi)=\sum_{k \in \mathbb{Z}^{n},|k|>N}\left|\hat{\psi}\left(B_{j}(\xi+k)\right)\right|^{2}, \quad R_{j}(\xi)=\sum_{k \in \mathbb{Z}^{n}}\left|\hat{\psi}\left(B_{j}(\xi+k)\right)\right|^{2} .
$$

Let $I_{n}=[-1 / 2,1 / 2)^{n}$ be the fundamental domain of $\mathbb{T}^{n}=\mathbb{R}^{n} / \mathbb{Z}^{n}$. We need to prove the following two lemmas.

Lemma 2.2. With the notation as above we have

$$
\lim _{j \rightarrow \infty}\left|\operatorname{det} B_{j}\right| R_{j}=c \quad \text { in the } L^{1}\left(I_{n}\right) \text { norm, where } c=\|\psi\|^{2} .
$$

Proof. Suppose that $g$ is a continuous function on $\mathbb{R}^{n}$ with compact support. Since $g$ is uniformly continuous, then a standard argument using the Riemann integral shows that

$$
\left|\operatorname{det} B_{j}\right| \sum_{k \in \mathbb{Z}^{n}}\left|g\left(B_{j}(\xi+k)\right)\right|^{2} \rightarrow\|g\|_{2}^{2} \quad \text { uniformly on } I_{n} \text { as } j \rightarrow \infty .
$$


This implies that (2.4) holds for $g$ in place of $\hat{\psi}$. For a general $\psi \in L^{2}\left(\mathbb{R}^{n}\right)$ and $\varepsilon>0$ find a continuous compactly supported $g$ such that ||$|g|^{2}-|\hat{\psi}|^{2} \|_{1}<\varepsilon$. Then,

$$
\begin{aligned}
& \left.\left|\operatorname{det} B_{j}\right| \int_{I_{n}}\left|R_{j}(\xi)-\sum_{k \in \mathbb{Z}^{n}}\right| g\left(B_{j}(\xi+k)\right)\right|^{2} \mid d \xi \\
& \leq\left.\sum_{k \in \mathbb{Z}^{n}}\left|\operatorname{det} B_{j}\right| \int_{I_{n}}|| \hat{\psi}\left(B_{j}(\xi+k)\right)\right|^{2}-\left|g\left(B_{j}(\xi+k)\right)\right|^{2}|d \xi=||g|^{2}-|\hat{\psi}|^{2} \|_{1}<\varepsilon
\end{aligned}
$$

Combining (2.5) and (2.6) yields (2.4).

Lemma 2.3. With the notation as above we have

$$
\limsup _{j \rightarrow \infty} L_{j}(\xi) / R_{j}(\xi)=1 \quad \text { for a.e. } \xi \in I_{n} .
$$

Here, we interpret expressions of the form $0 / 0$ as 0.

Proof. Fix $0<\delta<1$ and define the sets

$$
E_{j}=\left\{\xi \in I_{n}: L_{j}(\xi) \leq \delta R_{j}(\xi)\right\} .
$$

Then, by the Lebesgue Dominated Convergence Theorem and (1.9),

$$
\begin{aligned}
& \left|\operatorname{det} B_{j}\right| \int_{I_{n}} L_{j}(\xi) d \xi \geq\left|\operatorname{det} B_{j}\right| \int_{B(0, N+\sqrt{n})^{c}}\left|\hat{\psi}\left(B_{j} \xi\right)\right|^{2} d \xi \\
& =\int_{B_{j}\left(B(0, N+\sqrt{n})^{c}\right)}|\hat{\psi}(\xi)|^{2} d \xi \rightarrow\|\psi\|^{2}=\left|\operatorname{det} B_{j}\right| \int_{I_{n}} R_{j}(\xi) d \xi \quad \text { as } j \rightarrow \infty .
\end{aligned}
$$

In the first inequality above we have used a simple periodization argument and the fact that

$$
B(0, N+\sqrt{n}) \subset \sum_{|k| \leq N}\left(k+I_{n}\right) .
$$

Hence,

$$
\begin{aligned}
(1-\delta)\left|\operatorname{det} B_{j}\right| \int_{E_{j}} R_{j}(\xi) d \xi & \leq\left|\operatorname{det} B_{j}\right| \int_{E_{j}}\left(R_{j}(\xi)-L_{j}(\xi)\right) d \xi \\
& \leq\left|\operatorname{det} B_{j}\right| \int_{I_{n}}\left(R_{j}(\xi)-L_{j}(\xi)\right) d \xi \rightarrow 0 \quad \text { as } j \rightarrow \infty .
\end{aligned}
$$

By (2.4),

$$
|| \operatorname{det} B_{j}\left|\int_{E_{j}} R_{j}(\xi) d \xi-c\right| E_{j}|| \leq\left|\operatorname{det} B_{j}\right| \int_{I_{n}}\left|R_{j}(\xi)-c\right| d \xi \rightarrow 0 \quad \text { as } j \rightarrow \infty
$$

where $c=\|\psi\|^{2}$. Combining (2.8) and (2.9) yields

$$
\left|E_{j}\right| \rightarrow 0 \quad \text { as } j \rightarrow \infty \text {. }
$$

Since

we have that

$$
R_{j}(\xi) \geq L_{j}(\xi)>\delta R_{j}(\xi) \geq 0 \quad \text { for } \xi \in I_{n} \backslash E_{j}
$$

Hence, (2.10) yields

$$
L_{j}(\xi) / R_{j}(\xi)>\delta \quad \text { for } \xi \in I_{n} \backslash E_{j}
$$

$$
\limsup _{j \rightarrow \infty} L_{j}(\xi) / R_{j}(\xi) \geq \delta \quad \text { for a.e. } \xi \in I_{n} .
$$


Since $0<\delta<1$ is arbitrary, this proves (2.7) and completes the proof of the lemma.

We are now ready to finish the proof of Theorem 1.1, Lemma 2.3 and (2.2) imply that for any $N>0$,

$$
\limsup _{j \rightarrow \infty} \sum_{k \in \mathbb{Z}^{n},|k|>N} \sigma_{j}(\xi+k)=1 \quad \text { for a.e. } \xi \in I_{n} .
$$

Using (2.11) we shall construct inductively two strictly increasing sequences $\left\{j_{i}\right\}$ and $\left\{N_{i}\right\}$ of integers such that the sets

$$
S_{i}=\left\{\xi \in I_{n}: \sum_{k \in \mathbb{Z}^{n}, N_{i-1}<|k| \leq N_{i}} \sigma_{j_{i}}(\xi+k) \geq 1 / 2\right\}
$$

satisfy

$$
\left|I_{n} \backslash S_{i}\right|<2^{-i} \quad \text { for all } i \in \mathbb{N} .
$$

Indeed, let $N_{0}=0$. By (2.11) choose $j_{1}$ large enough so that

$$
\left|S_{1}^{\prime}\right|>1 / 2, \quad \text { where } S_{1}^{\prime}=\left\{\xi \in I_{n}: \sum_{k \in \mathbb{Z}^{n}, N_{0}<|k|} \sigma_{j_{1}}(\xi+k) \geq 3 / 4\right\} .
$$

Then, choose $N_{1}$ large enough so that the set $S_{1}$ given by (2.12) also satisfies $\left|S_{1}\right|>1 / 2$. Assuming that we have defined $j_{1}<\ldots<j_{r}$ and $N_{1}<\ldots<N_{r}$ for some $r \in \mathbb{N}$, choose $j_{r+1}$ so that

$$
\left|S_{r+1}^{\prime}\right|>1-2^{-r-1}, \quad \text { where } S_{r+1}^{\prime}=\left\{\xi \in I_{n}: \sum_{k \in \mathbb{Z}^{n}, N_{r}<|k|} \sigma_{j_{r+1}}(\xi+k) \geq 3 / 4\right\} .
$$

Then, choose $N_{r+1}$ large enough so that (2.13) holds for the set $S_{r+1}$ given by (2.12). This proves (2.13).

Finally, using (2.1) and (2.3) we have

$\operatorname{dim}_{V}(\xi)=\sum_{k \in \mathbb{Z}^{n}} \sigma_{V}(\xi+k) \geq \sum_{i=1}^{\infty} \sum_{\substack{k \in \mathbb{Z}^{n} \\ N_{i-1}<|k| \leq N_{i}}} \sigma_{j_{i}}(\xi+k) \geq \sum_{i=r}^{\infty} \frac{1}{2}=\infty$ for a.e. $\xi \in \tilde{S}_{r}$,

where

$$
\tilde{S}_{r}=\bigcap_{i=r}^{\infty} S_{i} \quad \text { for } r \in \mathbb{N} .
$$

By (2.13), $\left|I_{n} \backslash \tilde{S}_{r}\right| \leq 2^{-r+1} \rightarrow 0$ as $r \rightarrow \infty$. Hence,

$$
\operatorname{dim}_{V}(\xi)=\infty \quad \text { for a.e. } \xi \in I_{n},
$$

which completes the proof of Theorem 1.1

Remark 2.1. We remark that Theorem 1.1 is the optimal result of this type for general SI spaces. More precisely, if the only information provided to us about an SI space $V$ is its dimension function $\operatorname{dim}_{V}$, then one could not do better. That is, there are many examples of SI spaces with $\operatorname{dim}_{V} \equiv \infty$ where (1.10) fails. The most trivial example is $V=L^{2}\left(\mathbb{R}^{n}\right)$.

On the other hand, one can provide examples of SI spaces with $\operatorname{dim}_{V} \equiv \infty$ such that (1.10) holds. An example of this kind for $A_{j}$ of the form $A_{j}=A^{-j}$, where $A$ is an integer expansive matrix, follows from the characterization of dimension 
functions of GMRAs due to Rzeszotnik and the author [9]. Indeed, by [9, Theorem 3.6] there exists a GMRA $\left\{D^{j}(V)\right\}_{j \in \mathbb{Z}}$ such that $\operatorname{dim}_{V} \equiv \infty$. Consequently, $\bigcap_{j \in \mathbb{Z}} D^{j}(V)=\{0\}$.

More delicate examples of SI spaces which either fail or fulfill the intersection property (1.8) are shown in the next section.

\section{EXAMPLES}

In this section we give an example of two refinable SI spaces $V$ and $V^{\prime}$ sharing the same spectral functions, but which behave differently under dilations. Despite their close relationship, the space $V$ fails the intersection property, whereas the other space $V^{\prime}$ satisfies this property. A seemingly innocuous change in phases of generators, which is not detected by the spectral function, can lead to totally different outcomes. This further illustrates how delicate the intersection property (1.8) is. In light of Theorem 1.1 both spaces $V$ and $V^{\prime}$ have dimension functions constantly equal to $\infty$.

For simplicity, we restrict our attention to one dimension. Let $D$ be the dyadic dilation operator given by $D f(x)=\sqrt{2} f(2 x)$.

Example 3.1. Consider the set of generators $\mathcal{A}=\left\{\varphi_{i}: i=0,1, \ldots\right\}$ by

$$
\hat{\varphi}_{i}= \begin{cases}\mathbf{1}_{[0,1]} & i=0, \\ \mathbf{1}_{\left[2^{i-1}, 2^{i}\right]}, & i=1,2, \ldots\end{cases}
$$

Let $V=\mathcal{S}(\mathcal{A})$ be the corresponding SI space. We claim that $V$ is a refinable space and that its spectral function is given by

$$
\sigma_{V}=\mathbf{1}_{[0,1]}+\sum_{i=1}^{\infty} 2^{1-i} \mathbf{1}_{\left[2^{i-1}, 2^{i}\right]} .
$$

To see that $V$ is refinable note that $\varphi_{i+1}=\sqrt{2} D\left(\varphi_{i}\right)$ for $i=1,2, \ldots$. Moreover, $\varphi_{0}, \varphi_{1} \in D\left(\mathcal{S}\left(\varphi_{0}\right)\right)=D\left(\check{L}^{2}([0,1])\right)=\check{L}^{2}([0,2])$. Hence, $\mathcal{A} \subset D(V)$. Since $D(V)$ is SI, we have $V=\mathcal{S}(\mathcal{A}) \subset D(V)$. To see (3.1) we observe that

$$
\mathcal{S}\left(\varphi_{i}\right) \perp \mathcal{S}\left(\varphi_{j}\right) \quad \text { for } i \neq j .
$$

Moreover, for any $i=1,2, \ldots$,

$$
\sigma_{\mathcal{S}\left(\varphi_{i}\right)}(\xi)=\frac{\left|\hat{\varphi}_{i}(\xi)\right|^{2}}{\sum_{k \in \mathbb{Z}}\left|\hat{\varphi}_{i}(\xi+k)\right|^{2}}=2^{1-i} \quad \text { for } \xi \in \operatorname{supp} \hat{\varphi}_{i}=\left[2^{i-1}, 2^{i}\right],
$$

and $\sigma_{\mathcal{S}\left(\varphi_{i}\right)}(\xi)=0$ for $\xi \notin \operatorname{supp} \hat{\varphi}_{i}$. Thus, the countable additivity property of the spectral function

$$
\sigma_{V}=\sum_{i=0}^{\infty} \sigma_{\mathcal{S}\left(\varphi_{i}\right)}
$$

implies (3.1). Despite that the spectral function $\sigma_{V}$ vanishes at infinity, the intersection property fails, i.e.,

$$
\bigcap_{j=0}^{\infty} D^{-j}(V) \neq\{0\} .
$$

Namely, the generators $\varphi_{i}, i=1,2, \ldots$, lie in this intersection. Indeed, $\varphi_{i}=$ $2^{-j / 2} D^{-j}\left(\varphi_{i+j}\right)$ for $j=0,1, \ldots$ and $i=1,2, \ldots$ 
Next, we will modify this example to produce another refinable SI space $V^{\prime}$ which has the same spectral function as $V$, but the intersection property holds, i.e.,

$$
\bigcap_{j=0}^{\infty} D^{-j}\left(V^{\prime}\right)=\{0\}
$$

Example 3.2. Define the function $\Pi: \mathbb{R} \rightarrow\{-1,1\}$ by

$$
\Pi(\xi)=\prod_{j=-1}^{\infty} p\left(2^{-j} \xi\right), \quad \text { where } p(\xi)= \begin{cases}1 & \text { if }\lfloor\xi\rfloor \text { is even, } \\ -1 & \text { otherwise. }\end{cases}
$$

One can show that the function $\Pi(\xi), \xi>0$, can also be described as follows: if the number of 1's in the binary expansion of $\lfloor 2 \xi\rfloor$ is even, then $\Pi(\xi)=1$. Otherwise, $\Pi(\xi)=-1$.

Consider the set of generators $\mathcal{A}^{\prime}=\left\{\phi_{i}: i=0,1, \ldots\right\}$ defined by

$$
\hat{\phi}_{i}(\xi)=\Pi(\xi) \hat{\varphi}_{i}(\xi)
$$

Let $V^{\prime}=\mathcal{S}\left(\mathcal{A}^{\prime}\right)$ be the corresponding SI space. Clearly, the spectral functions of the spaces $\mathcal{S}\left(\varphi_{i}\right)$ and $\mathcal{S}\left(\phi_{i}\right)$ are the same. Since the supports of the $\hat{\phi}_{i}$ 's are mutually disjoint, the spaces $\mathcal{S}\left(\phi_{i}\right)$ are mutually orthogonal. Hence, (3.3) and (3.4) yield

$$
\sigma_{V^{\prime}}=\sigma_{V}=\mathbf{1}_{[0,1]}+\sum_{i=1}^{\infty} 2^{-i+1} \mathbf{1}_{\left[2^{i-1}, 2^{i}\right]} .
$$

To see that $V^{\prime}$ is refinable note that for $i=1,2, \ldots$,

$$
\hat{\phi}_{i+1}(\xi)=\Pi(\xi) \hat{\varphi}_{i+1}(\xi)=p(2 \xi) \Pi(\xi / 2) \hat{\varphi}_{i}(\xi / 2)=p(2 \xi) \hat{\phi}_{i}(\xi / 2) .
$$

Since $\xi \mapsto p(2 \xi)$ is $\mathbb{Z}$-periodic, $\phi_{i+1} \in \mathcal{S}\left(D \phi_{i}\right)$ by Lemma 2.1. Likewise, $D \phi_{i} \in$ $\mathcal{S}\left(\phi_{i+1}\right)$, which implies that $\mathcal{S}\left(\phi_{i+1}\right)=\mathcal{S}\left(D \phi_{i}\right)$. Since $T_{k} D=D T_{2 k}$, we have

$$
\mathcal{S}\left(\phi_{i+1}\right)=\mathcal{S}\left(D \phi_{i}\right) \subset D\left(\mathcal{S}\left(\phi_{i}\right)\right) \quad \text { for } i=1,2, \ldots
$$

Moreover, $\mathcal{S}\left(\phi_{0}\right), \mathcal{S}\left(\phi_{1}\right) \subset \check{L}^{2}([0,2])=D\left(\check{L}^{2}([0,1])\right)=D\left(\mathcal{S}\left(\phi_{0}\right)\right)$. Hence, $V^{\prime} \subset$ $D\left(V^{\prime}\right)$.

Finally, to establish the intersection property (3.5) we first observe that

$$
V^{\prime}=\mathcal{S}\left(\phi_{0}\right) \oplus\left(\bigoplus_{j=1}^{\infty} \mathcal{S}\left(\phi_{i}\right)\right)=\left(\bigoplus_{j=-\infty}^{-1} D^{j}\left(\mathcal{S}\left(\phi_{1}\right)\right)\right) \oplus\left(\bigoplus_{j=1}^{\infty} \mathcal{S}\left(\phi_{j}\right)\right) .
$$

Indeed, the above decomposition follows immediately from $\mathcal{S}\left(\phi_{0}\right)=\check{L}^{2}([0,1])$ and $\mathcal{S}\left(\phi_{1}\right)=\check{L}^{2}([1,2])$. By (3.8), the dilation operators $D^{-l}, l \in \mathbb{N}$, shift the components of (3.9) by $l$ terms, i.e.,

$$
\begin{array}{rlrl}
D^{-l}\left(D^{j}\left(\mathcal{S}\left(\phi_{1}\right)\right)\right) & =D^{j-l}\left(\mathcal{S}\left(\phi_{1}\right)\right) & & \text { for }-\infty<j \leq-1, \\
D^{-l}\left(\mathcal{S}\left(\phi_{j}\right)\right) & \subset D^{j-l}\left(\mathcal{S}\left(\phi_{1}\right)\right) & \text { for } 1 \leq j \leq l, \\
D^{-l}\left(\mathcal{S}\left(\phi_{j}\right)\right) & \subset \mathcal{S}\left(\phi_{j-l}\right) & \text { for } l+1 \leq j<\infty .
\end{array}
$$

Therefore,

$$
\bigcap_{i=0}^{\infty} D^{-i}\left(V^{\prime}\right)=\bigoplus_{j \in \mathbb{Z} \backslash\{0\}} Z_{j}
$$


where

$$
Z_{j}= \begin{cases}D^{j}\left(\mathcal{S}\left(\phi_{1}\right)\right) \cap D^{j-1}\left(\mathcal{S}\left(\phi_{2}\right)\right) \cap D^{j-2}\left(\mathcal{S}\left(\phi_{3}\right)\right) \cap \ldots & \text { for }-\infty<j \leq-1, \\ \mathcal{S}\left(\phi_{j}\right) \cap D^{-1}\left(\mathcal{S}\left(\phi_{j+1}\right)\right) \cap D^{-2}\left(\mathcal{S}\left(\phi_{j+2}\right)\right) \cap \ldots & \text { for } 1 \leq j<\infty\end{cases}
$$

To complete the proof we must show that $Z_{j}=\{0\}$ for all $j \in \mathbb{Z} \backslash\{0\}$. A quick inspection of (3.10) shows that this is equivalent to

$$
\bigcap_{i=0}^{\infty} D^{-i}\left(\mathcal{S}\left(\phi_{j+i}\right)\right)=\{0\} \quad \text { for } 1 \leq j<\infty .
$$

Fix some $1 \leq j<\infty$. By Lemma 2.1 any $f \in \mathcal{S}\left(\phi_{j}\right)$ can be written as

$$
\hat{f}(\xi)=m_{0}(\xi) \hat{\phi}_{j}(\xi), \quad \text { for a.e. } \xi,
$$

for some measurable $\mathbb{Z}$-periodic $m_{0}$. The fact that $\left|\hat{\phi}_{j}\right|=\mathbf{1}_{\left[2^{j-1}, 2^{j}\right]}$ implies that $m_{0}$ is square-integrable over its period. Likewise, any $f \in \mathcal{D}^{-i}\left(\mathcal{S}\left(\phi_{j+i}\right)\right)$ can be written as

$$
\hat{f}(\xi)=m_{i}\left(2^{i} \xi\right) \hat{\phi}_{j+i}\left(2^{i} \xi\right), \quad \text { for a.e. } \xi,
$$

for some $m_{i} \in L^{2}(\mathbb{R} / \mathbb{Z})$. By (3.7),

$$
\hat{\phi}_{j+i}\left(2^{i} \xi\right)=p\left(2^{i+1} \xi\right) \hat{\phi}_{j+i-1}\left(2^{i-1} \xi\right)=\ldots=p\left(2^{i+1} \xi\right) p\left(2^{i} \xi\right) \cdot \ldots \cdot p\left(2^{2} \xi\right) \hat{\phi}_{j}(\xi) .
$$

Combining the above yields

$$
m_{0}(\xi)=m_{i}\left(2^{i} \xi\right) p\left(2^{i+1} \xi\right) p\left(2^{i} \xi\right) \cdot \ldots \cdot p\left(2^{2} \xi\right) \quad \text { for a.e. } \xi .
$$

Let $K$ be any dyadic interval of length $2^{-i}$, that is, $K=\left[k 2^{-i},(k+1) 2^{-i}\right]$ for some $k \in \mathbb{Z}$. Since $\xi \mapsto p\left(2^{i+1} \xi\right) m_{i}\left(2^{i} \xi\right)$ is $2^{-i} \mathbb{Z}$-periodic and $s(\xi)=p\left(2^{i} \xi\right) \cdot \ldots \cdot p\left(2^{2} \xi\right)$ is constant on dyadic intervals of length $2^{-i}$, we have

$$
\begin{aligned}
\int_{K} m_{0}(\xi) d \xi=\int_{K} p\left(2^{i+1} \xi\right) m_{i}\left(2^{i} \xi\right) s(\xi) d \xi & =c_{i} \int_{K} s(\xi) d \xi \\
& \text { where } c_{i}=\int_{0}^{2^{-i}} p\left(2^{i+1} \xi\right) m_{i}\left(2^{i} \xi\right) d \xi .
\end{aligned}
$$

Since the function $s$ takes only values \pm 1 , we have that

$$
\frac{1}{|K|} \int_{K} m_{0}(\xi) d \xi= \pm c_{i} \quad \text { for all dyadic intervals with }|K|=2^{-i} \text {. }
$$

While the function $s$ is constant on dyadic intervals of length $2^{-i}$, it takes two opposite values \pm 1 on any dyadic interval $K^{\prime}$ of length $2^{-i+1}$ due to the factor $p\left(2^{i} \xi\right)$. Hence, $\int_{K^{\prime}} m_{0}(\xi) d \xi=0$ for all dyadic intervals $K^{\prime}$ of length $2^{-i+1}$. Consequently, if a function $f$ belongs to the intersection (3.11), then the corresponding multiplier $m_{0}$ from (3.12) satisfies

$$
\int_{K} m_{0}(\xi) d \xi=0 \quad \text { for all dyadic intervals } K \text {. }
$$

By the Lebesgue Differentiation Theorem, $m_{0}(\xi)=0$ for a.e. $\xi$, and hence $f=0$, which proves (3.11). 
Finally, we note that the spaces $V$ and $V^{\prime}$ satisfy

$$
\overline{\bigcup_{j \in \mathbb{Z}} D^{j}(V)}=\overline{\bigcup_{j \in \mathbb{Z}} D^{j}\left(V^{\prime}\right)}=\check{L}^{2}(0, \infty) .
$$

Hence, if we add an extra generator $\varphi_{-1}$, where $\hat{\varphi}_{-1}=\mathbf{1}_{[-1,0]}$, then the SI spaces $V_{0}=V \oplus \mathcal{S}\left(\varphi_{-1}\right)$ and $V_{0}^{\prime}=V^{\prime} \oplus \mathcal{S}\left(\varphi_{-1}\right)$ will also satisfy the density property (1.4). Consequently, the sequence $V_{j}^{\prime}=D^{j}\left(V_{0}^{\prime}\right), j \in \mathbb{Z}$, forms a GMRA, whereas $V_{j}=D^{j}\left(V_{0}\right), j \in \mathbb{Z}$, is not a GMRA due to the failure of the intersection property (1.3). This is despite the fact that the core spaces $V_{0}$ and $V_{0}^{\prime}$ share the same spectral function.

\section{REFERENCES}

1. P. Auscher, Solution of two problems on wavelets, J. Geom. Anal. 5 (1995), 181-236. MR.1341029 (96g:42016)

2. L. Baggett, An abstract interpretation of the wavelet dimension function using group representations, J. Funct. Anal. 173 (2000), 1-20. MR.1760275 (2001j:42028)

3. L. Baggett, H. Medina, K. Merrill, Generalized multi-resolution analyses and a construction procedure for all wavelet sets in $\mathbb{R}^{n}$, J. Fourier Anal. Appl. 5 (1999), 563-573. MR1752590 (2001f:42055)

4. C. de Boor, R. DeVore, A. Ron, On the construction of multivariate (pre)wavelets, Constr. Approx. 9 (1993), 123-166. MR1215767 (94k:41048)

5. C. de Boor, R. DeVore, A. Ron, The structure of finitely generated shift-invariant spaces in $L_{2}\left(\mathbb{R}^{d}\right)$, J. Funct. Anal. 119 (1994), 37-78. MR1255273 (95g:46050)

6. M. Bownik, The structure of shift-invariant subspaces of $L^{2}\left(\mathbb{R}^{n}\right)$, J. Funct. Anal. $177(2000)$, 282-309. MR1795633 (2001k:42037)

7. M. Bownik, Riesz wavelets and generalized multiresolution analyses, Appl. Comput. Harmon. Anal. 14 (2003), 181-194. MR1984546 (2004d:42057)

8. M. Bownik, Baggett's problem for frame wavelets, Representations, Wavelets, and Frames: A Celebration of the Mathematical Work of Lawrence W. Baggett, Birkhäuser, 2008, pp. 153173.

9. M. Bownik, Z. Rzeszotnik, The spectral function of shift-invariant spaces, Michigan Math. J. 51 (2003), 387-414. MR1992954 (2004d:42058)

10. M. Bownik, Z. Rzeszotnik, On the existence of multiresolution analysis for framelets, Math. Ann. 332 (2005), 705-720. MR.2179772 (2006e:42051)

11. Q. Gu, D. Han, Frames, modular functions for shift-invariant subspaces and FMRA wavelet frames, Proc. Amer. Math. Soc. 133 (2005), 815-825. MR2113932 (2006b:42052)

12. H. Helson, Lectures on invariant subspaces, Academic Press, New York-London, 1964. MR.0171178 (30:1409)

13. E. Hernández, G. Weiss, A first course on wavelets, Studies in Advanced Mathematics, CRC Press, Boca Raton, FL, 1996. MR 1408902 (97i:42015)

14. S. Mallat, Multiresolution approximations and wavelet orthonormal bases of $L^{2}(\mathbb{R})$, Trans. Amer. Math. Soc. 315 (1989), 69-87. MR1008470 (90e:42046)

15. Z. Rzeszotnik, Calderón's condition and wavelets, Collect. Math. 52 (2001), 181-191. MR:1852037 (2002j:42039)

Department of Mathematics, University of Oregon, Eugene, Oregon 97403-1222

E-mail address: mbownik@uoregon.edu 